PROCEEDINGS OF THE

AMERICAN MATHEMATICAL SOCIETY

Volume 125, Number 5, May 1997, Pages 1511-1515

S 0002-9939(97)03788-X

\title{
FIXED POINTS OF THE MAPPING CLASS GROUP IN THE $S U(n)$ MODULI SPACES
}

\author{
JØRGEN ELLEGAARD ANDERSEN
}

(Communicated by Ronald Stern)

\begin{abstract}
Let $\Sigma$ be a compact oriented surface with or without boundary components. In this note we prove that if $\chi(\Sigma)<0$ then there exist infinitely many integers $n$ such that there is a point in the moduli space of irreducible flat $S U(n)$ connections on $\Sigma$ which is fixed by any orientation preserving diffeomorphism of $\Sigma$. Secondly we prove that for each orientation preserving diffeomorphism $f$ of $\Sigma$ and each $n \geq 2$ there is some $m$ such that $f$ has a fixed point in the moduli space of irreducible flat $S U\left(n^{m}\right)$ connections on $\Sigma$. Thirdly we prove that for all $n \geq 2$ there exists an integer $m$ such that the $m$ 'th power of any diffeomorphism fixes a certain point in the moduli space of irreducible flat $S U(n)$ connections on $\Sigma$.
\end{abstract}

\section{INTRODUCTION}

Let $\Sigma$ be an oriented compact surface with or without boundary components. Consider the fundamental group of the surface, $\pi_{1}$. Let $M(n)$ be the moduli space of irreducible flat $S U(n)$-connections on $\Sigma$. Hence we have that

$$
M(n)=\operatorname{Hom}^{\mathrm{irr}}\left(\pi_{1}, S U(n)\right) / S U(n) .
$$

Let $\Gamma$ be the mapping class group of $\Sigma$. Let $f$ be an element of $\Gamma$. Now $f$ acts on $M(n)$. We are interested in the following questions:

1. Which $f$ 's have fixed points in $M(n)$ ?

2. Does $\Gamma$ have a fixed point in $M(n)$ ?

We shall for certain $n$ answer these questions affirmatively. One should remark that the fixed point set of any finite order or reducible finite order diffeomorphism can be described explicitly (see [1]). The first question is therefore only interesting for pseudo-Anosov diffeomorphisms. Answering yes to 2 for all $n$ of course implies 1. It is however not quite clear if the techniques presented in this paper can establish that, though we can answer 1 positively for some nontrivial subgroups of $\Gamma$ for all $n$.

We shall here analyse this problem using elementary finite group theory. We will show that for all primes $p$ greater than 5 there is an integer $m$ such that $\Gamma$ has a fixed point in $M\left(p^{m}\right)$. We will also see that our technique can show that for all

Received by the editors November 17, 1995.

1991 Mathematics Subject Classification. Primary 53C07.

Supported in part by NSF grant DMS-93-09653, while the author was visiting the University of California, Berkeley.

(C)1997 American Mathematical Society 
$n \geq 2$ there is some integer $m$ (which may depend on $n$ ) and a point in $M(n)$ which is preserved by the $m$ 'th power of any $f$ in $\Gamma$.

In some particular cases, a positive answer to question 1 was obtained by Frohman in [2] (see also [3]). By using some involved cohomology calculations on the classifying space of certain gauge groups, Frohman gives an existence proof for fixed points for certain diffeomorphism of surfaces with 1 boundary component.

The author would like to thank both Prof. W. Thurston and Prof. G. Bergman for helpful discussions on this subject.

\section{INVARIANT NORMAL SUBGROUPS \\ OF THE FUNDAMENTAL GROUP OF A SURFACE}

Let $\Sigma$ be a compact oriented surface with or without boundary components. Consider the fundamental group of the surface, $\pi_{1}=\pi_{1}(\Sigma)$. Suppose $f$ is an orientation preserving diffeomorphism of $\Sigma$. We are now interested in $f$-invariant normal finite index subgroups of $\pi_{1}$. Suppose $\pi$ is a normal subgroup of finite index of $\pi_{1}$. Then $f(\pi)$ is again a normal subgroup of the same finite index as $\pi$. Let $\tilde{\pi}$ be the intersection

$$
\tilde{\pi}=\bigcap_{n} f^{n}(\pi)
$$

This intersection is actually a finite intersection, since there are only finitely many normal subgroups of a given index. In fact, the number of subgroups of index $i$ of $\pi_{1}$ is bounded by the number of $i$-fold covers of $\Sigma$. Let $m$ be the smallest integer such that

$$
\tilde{\pi}=\bigcap_{n=0}^{m-1} f^{n}(\pi)
$$

Hence we have a finite quotient group $G_{f}(\pi)=\pi_{1} / \tilde{\pi}$, on which there is an induced action of $f$. If we let

$$
\tilde{\pi}_{k}=\bigcap_{n=0}^{k} f^{n}(\pi),
$$

then we have the following diagram:




We have that $\left(q_{1}, q_{2}\right)$ injects $\pi_{1} / \tilde{\pi}_{k+1}$ as a subgroup of $\pi_{1} / \tilde{\pi}_{k} \times \pi_{1} / f^{k+1}(\pi)$. Moreover, we also see that $\tilde{\pi}_{k} / \tilde{\pi}_{k+1}$ injects as a normal subgroup of $\pi_{1} / f^{k+1}(\pi)$, and $f$ induces an isomorphism of $\pi_{1} / f^{k+1}(\pi)$ with $\pi_{1} / \pi$.

Now in order to be able to control the representation theory of $\pi_{1} / \tilde{\pi}$, we are going to assume that $\pi_{1} / \pi$ is a simple group. We will see shortly that there are lots of examples where this is the case. The reason for this assumption is clear from the following lemma.

Lemma 2.1. In the case where $\pi_{1} / \pi$ is a simple group we have that

$$
\pi_{1} / \tilde{\pi} \cong \pi_{1} / \pi \times \pi_{1} / \pi \times \ldots \times \pi_{1} / \pi \text { (m factors). }
$$

Proof. We shall prove the lemma by induction. Assume that

$$
\left.\pi_{1} / \tilde{\pi}_{k} \cong \pi_{1} / \pi \times \pi_{1} / \pi \times \ldots \times \pi_{1} / \pi \text { ( } k+1 \text { factors }\right) .
$$

This assumption is clearly true when $k=0$. Since $\tilde{\pi}_{k} / \tilde{\pi}_{k+1}$ is a normal subgroup of $\pi_{1} / f^{k+1}(\pi)$, which is isomorphic to $\pi_{1} / \pi$, we have that $\tilde{\pi}_{k} / \tilde{\pi}_{k+1}$ must be either trivial or isomorphic to $\pi_{1} / \pi$. If $\tilde{\pi}_{k} / \tilde{\pi}_{k+1}$ is trivial, then $\tilde{\pi}_{k}=\tilde{\pi}$. So $k=m-1$, in which case we have proved the lemma. Assume then that $\tilde{\pi}_{k} / \tilde{\pi}_{k+1} \cong \pi_{1} / \pi$. From the vertical sequence in the above diagram, we then see that $\pi_{1} / \tilde{\pi}_{k+1}$ has order $\left|\pi_{1} / \pi\right|^{k+1} \times\left|\pi_{1} / \pi\right|=\left|\pi_{1} / \pi\right|^{k+2}$. But then $\left(q_{1}, q_{2}\right)$ must be an isomorphism from $\pi_{1} / \tilde{\pi}_{k+1}$ to $\pi_{1} / \tilde{\pi}_{k} \times \pi_{1} / f^{k+1}(\pi)$. By induction we then get that

$$
\left.\pi_{1} / \tilde{\pi}_{k+1} \cong \pi_{1} / \pi \times \pi_{1} / \pi \times \ldots \times \pi_{1} / \pi \text { ( } k+2 \text { factors }\right) .
$$

Remark. We note that the $m$ in the above lemma is clearly bounded by the number of principal $\pi_{1} / \pi$ bundles over $\Sigma$, which in turn is bounded by the number of $\left|\pi_{1} / \pi\right|-$ sheeted covers of $\Sigma$.

Notice that in the above discussion, we could have defined

$$
\bar{\pi}=\bigcap_{f \in \Gamma} f(\pi) .
$$

Exactly the same arguments show that there is some $m$ such that

$$
\left.\pi_{1} / \bar{\pi} \cong \pi_{1} / \pi \times \pi_{1} / \pi \times \ldots \times \pi_{1} / \pi \text { ( } m \text { factors }\right) .
$$

This way, we get a finite group $G_{\Gamma}(\pi)=\pi_{1} / \bar{\pi}$, on which there is an induced action of the mapping class group $\Gamma$.

Using the notation from the introduction, we see that in order to construct a fixed point for $\Gamma$ 's action on $M(n)$, we just need to find an irreducible $n$-dimensional representation of the finite group $G_{\Gamma}(\pi)$, which is invariant under $\Gamma$. One way to construct such a situation without knowing anything about how $\Gamma$ acts on $G_{\Gamma}(\pi)$, is to find a dimension in which $G_{\Gamma}(\pi)$ has only one irreducible representation. Suppose we can find a normal subgroup $\pi$ of $\pi_{1}$ such that $\pi_{1} / \pi$ is simple and has a unique irreducible representation in dimension $p$, where $p$ is a prime, and such that $\pi_{1} / \pi$ does not have any other irreducible representations of dimension $p^{n}$ for any $n$. Then $G_{\Gamma}(\pi)$ will have a unique irreducible representation of dimension $p^{m}$. Hence we see that $\Gamma$ will fix that corresponding point in $M\left(p^{m}\right)$.

It is now time to deliver on the promised normal subgroups.

Consider the alternating groups $A_{d}, d \geq 5$. These are all simple and can be given a presentation using two generators (see e.g. [5]). Since the free group on 
two generators is the quotient of the fundamental group of any oriented surface of negative Euler-characteristic, we see there is a normal subgroup $\pi^{d}$ of $\pi_{1}$ such that

$$
A_{d}=\pi_{1} / \pi^{d} .
$$

Consider now the case where $d=p+1$, where $p>5$ is a prime. We then have the standard irreducible representation $V$ of $A_{d}$ of dimension $p$. Moreover, it is easy to prove that this is the only representation of dimension $p$ (see e.g. p.67 in [4]). ${ }^{1} \mathrm{By}$ using the hook length formula one can very easily prove that the symmetric group $S_{d}$ does not have any irreducible representations of dimension $p^{n}$ or $2 p^{n}$ for $n>1$. If it did, we would have that

$$
p^{n}=\frac{(p+1) !}{h_{1}} \text { or } 2 p^{n}=\frac{(p+1) !}{h_{2}},
$$

where here $h_{1}, h_{2} \in \mathbb{N}$ are the hook lengths of the representations. But from this and unique factorization we get that $n=1$. Hence $A_{d}$ cannot have any irreducible representations of dimension $p^{n}$ for $n>1$. (See Proposition 5.1 p.64 in [4] for the relation between the representations of $A_{d}$ and $S_{d}$.)

Hence $\left(\pi^{d}, A_{d}, V\right)$ satisfies all the requirements to guarantee that there is an $m=m(d)$ which is bounded by the number of $\frac{d !}{2}$-sheeted covers of $\Sigma$, such that $\Gamma$ has a fixed point in $M\left(p^{m}\right)$.

Hence we have proved

Theorem 2.1. For any prime $p>5$ there is an $m=m(p)$ such that $\Gamma$ has a fixed point in $M\left(p^{m}\right)$.

Now, in the case where we are just interested in one element $f \in \Gamma$, we can actually say a bit more. From the proof of lemma 2.1 we get in fact an $f$ equivariant identification

$$
G_{f}(\pi) \cong \pi_{1} / \pi \times \pi_{1} / f(\pi) \times \ldots \times \pi_{1} / f^{m-1}(\pi),
$$

which means that when we use the identification

$$
G_{f}(\pi) \cong \pi_{1} / \pi \times \pi_{1} / \pi \times \ldots \times \pi_{1} / \pi(m \text { factors }),
$$

$f$ just becomes

$$
f\left(g_{1}, \ldots, g_{m}\right)=\left(f^{m}\left(g_{m}\right), g_{1}, g_{2}, \ldots, g_{m-1}\right) .
$$

Hence, if we have an irreducible $n$-dimensional representation $\rho$ of $\pi_{1} / \pi$ whose isomorphism class is preserved by $f^{m}$, then the isomorphism class of $\rho_{G_{f}}=\rho \otimes \ldots \otimes \rho$ is preserved by $f$. If $\rho$ is the only irreducible representation of $\pi_{1} / \pi$ of dimension $n$, the isomorphism class of $\rho$ must be preserved by $f^{m}$.

To construct some concrete examples, consider the normal subgroup $\pi^{d}$ of $\pi_{1}$ considered above. Now $d$ is any integer greather than or equal to 5 , but not equal to 6. As we mentioned before, there is only one irreducible representation of dimension $n=d-1$ of $A_{d}$. Let $\rho_{n}$ be that representation. $\rho_{G_{n}}=\rho_{n} \otimes \ldots \otimes \rho_{n}$ then gives a fixed point for $f$ in $M\left(n^{m}\right)$.

We can alternatively fix the normal subgroup $\pi$ of $\pi_{1}$ and then change $f$ to a power of $f$. We no longer have to worry about what $f$ maps $\pi$ to, since we will simply replace $f$ by a power of $f$ which fixes $\pi$. The requirement that $\pi_{1} / \pi$ should

\footnotetext{
${ }^{1}$ This is not the case for $A_{6}$, so we have excluded this case.
} 
be simple is no longer needed. We can for example make the following construction. Let $\pi$ be a normal subgroup of $\pi_{1}$ such that

$$
\pi_{1} / \pi=S_{3} .
$$

We then know that for some $m$ less than the number of 6 -sheeted covers of $\Sigma$, we have

$$
f^{m}(\pi)=(\pi)
$$

Now $S_{3}$ has a unique unitary irreducible 2-dimensional representation. Then this reprentation gives us a point in $M(2)$ which is fixed by $f^{m}$ for all $f \in \Gamma$. (We have here used the same symbol $M(2)$ to represent the $U(2)$ and the $S U(2)$ moduli space.) The smallest $m$ that does this will of course depend on $f$. However, if we let $l$ be the number of normal subgroups of $\pi_{1}$ whose quotient is $S_{3}$, then $f^{l}$ preserves the given point in $M(2)$.

Now notice that each $f$ in $\Gamma$ permutes the $l$ normal subgroups with $S_{3}$ quotient; hence we get a homomorphism from $\Gamma$ to $S_{l}$. The kernel of this homomorphism is of course a normal subgroup of finite index of $\Gamma$. For the elements $f$ in this normal subgroup, we can by definition take $m=1$ above, i.e. they fix the above described point in $M(2)$.

To get such fixed points in the moduli spaces $M(n)$ where $n$ is not 2 , we just repeat the above construction, where we replace $S_{3}$ with some group which has a unique irreducible representation in dimension $n$. For $n \neq 5$ just take the unique irreducible representation of $A_{n+1}$ of dimension $n$. For $n=5$ take the unique irreducible 5-dimensional representation of $A_{4}$.

\section{REFERENCES}

[1] J. E. Andersen, The Witten Invariant of finite order mapping tori I., University of Aarhus, Department of Mathematics Preprint (1995-21)

[2] C.D. Frohman, Unitary Representations of knot groups, Topology 32 (1993) 121-144 MR 94g:57003

[3] C.D. Frohman \& D.D. Long, Casson's invariant and surgery on knots, Procedings of the Edinburg Mathmatical Society 35 (1992) 383-395 MR 94a:57012

[4] W. Fulton \& J. Harris, Representation Theory, A first course., Springer Graduate Texts in Mathematics, Springer-Verlag, 1991 MR 93a:20069

[5] S. Lang, Algebra, Addison-Wesley 1984. MR 86j:00003

Department of Mathematics, University of Aarhus, DK-8000 Aarhus, Denmark

E-mail address: andersen@mi.aau.dk

Current address: Mathematical Sciences Research Institute, Berkeley, California 94720 